\title{
Kernos
}

Revue internationale et pluridisciplinaire de religion grecque antique

$17 \mid 2004$

Varia

\section{Walter BURKERT, Die Griechen und der Orient. Von Homer bis zu den Magiern}

\section{Vinciane Pirenne-Delforge}

URL : http://journals.openedition.org/kernos/1485

DOI : 10.4000/kernos. 1485

ISSN : 2034-7871

\section{Éditeur}

Centre international d'étude de la religion grecque antique

\section{Édition imprimée}

Date de publication : 1 janvier 2004

ISSN : 0776-3824

\section{Référence électronique}

Vinciane Pirenne-Delforge, « Walter BURKERT, Die Griechen und der Orient. Von Homer bis zu den Magiern », Kernos [En ligne], 17 | 2004, mis en ligne le 22 avril 2011, consulté le 24 septembre 2020 URL : http://journals.openedition.org/kernos/1485 ; DOI : https://doi.org/10.4000/kernos.1485 
d'Iphiklès au chant XI, guérison des Proetides au chant XV). L'évocation de ce personnage, qui en XI, 291 n'est même pas nommé, présuppose l'existence d'une tradition ancienne le concernant que E. Pellizer suit depuis l'Odyssée jusqu'à Bacchylicle. Plusieurs articles adoptent également une approche comparative. Parmi ceux-ci, nous voudrions souligner l'intéressant éclairage du folkloriste J. Abry sur les ruses mises en ouvres par Ulysse dans sa confrontation avec Polyphème. Au-delà d'une forme parfois déconcertante, cette communication résolument multidisciplinaire met remarquablement en perspective la naïveté du cyclope et sa totale incapacité à affronter les jeux langagiers du héros. B. Sergent quant à lui s'attache à démontrer que, si les Phéaciens sont bien, comme cela a été souligné mainte fois, des passeurs vers le monde des morts, leur pays correspond également à une vision grecque et indoeuropéenne des enfers. Cette image de Schérie comporterait de nombreux traits mycéniens.

Dans cet aperçu trop rapide d'un ouvrage si foisonnant nous avons dû faire une sélection, et donc laisser de côté des études de valeur dans lesquelles homérisants et spécialistes d'histoire littétaire se plongeront avec profit. Des index très utiles et des résumés des communications permettent une orientation aisée dans un volume forcément disparate en raison de la diversité des thèmes abordés.

O. Gengler

(Paris - EHESS)

BURKERT Walter, Die Griechen und der Orient. Von Homer bis zu den Magiern, aus dem Italienischen ins Deutsche übertragen vom Verfasser, München, Beck, 2003. 1 vol. $12,5 \times 20,5$ cm, 176 p. ISBN : 3-406-50247-4.

L'origine de cet ouvrage remonte à une série de leçons données à l'Université de Venise en avril 1996. Trois ans plus tard paraissait un petit ouvrage en italien, intitulé Da Omero ai Magi. La tradizione orientale nella cultura greca, qui a donné lieu à une traduction française (La tradition orientale dans la culture grecque, Paris, 2001) et espagnole (De Homero a los Magos, Barcelone, 2002). Voici maintenant une version allemande, qui n'est pas une traduction à proprement parler, mais bien un nouveau livre, retravaillé et augmenté par l'A. lui-même autour des thèmes dont on sait qu'ils lui sont chers. Faut-il rappeler son travail pionnier en la matière, Die orientalisierende Epoche in der griechischen Religion und Literatur (Heidelberg, 1984)?

Une nouvelle introduction repose globalement le problème de l'arrière-plan oriental de la Grèce classique - depuis la période mycénienne - et des contacts intercultulrels qui mettent à mal la vision d'un prétendu « miracle grec » dont l'A. retrace brièvement l'historiographie, depuis la Renaissance jusqu'aux coups de boutoir de la Black Athena de Martin Bernal. Le premier chapitre, très court, aborde la question de l'adoption et de l'adaptation de l'alphabet à des fins manifestement littéraires au départ. Les deux chapitres suivants remontent aux leçons vénitiennes. L'un propose un tel faisceau de convergences entre les récits épiques grecs et mésopotamiens (déjà largement présent dans Die orientalisierende Epoche et sal traduction anglaise The Oriental Revolution [1992]) qu'il est désormais vain de résister au constat des influences subies par le matériau épique grec, même si la question des canaux et des modalités de cette transmission reste largement ouverte (rituel, iconographique, littéraire). L'autre chapitre relativise tout autant l'originalité de la première pensée philosophique grecque en la mettant en regard des textes cosmogoniques et sapientaux proche-orientaux. La quatrième partie place sous le titre Orpheus und Ägypten ce qui apparaissait sous celui de L'orfismo riscoperto dans la version originale. Le cour du propos est le même et fait 
état de la révolution que les découvertes des trente dernières années ont suscitée dans l'interprétation de l'orphisme et des mystères bachiques. On ne peut que saluer la clarté, l'actualité et l'équilibre du bilan qui est ainsi fourni sur ce sujet complexe ( $c f$. aussi le numéro spécial de la Revue de l'Histoire des Religions 219/4 [2002]). L'A. a manifestement souhaité orienter davantage ses développements antérieurs - qui faisaient déjà place à l'Égypte - du côté d'une influence égyptienne en les insérant entre une introduction et une conclusion qui insistent sur le témoignage d'Hérodote. La dernière partie touche aux relations entre la Grèce et la Perse avec la figure des magol. Une introduction aux problèmes particuliers posés par la tradition littéraire et spirituelle iranienne et zoroastrienne est suivie d'une présentation des textes grecs surtout philosophiques - qui laissent percevoir l'empreinte de rituels et de spéculations relatives aux mages iraniens, notamment dans les théories qui concernent les âmes, les démons et les conceptions de l'au-delà. L'apparition des magoi dans une colonne nouvellement déchiffrée du papyrus de Derveni donne aux réflexions de l'A. une pertinence remarquable.

Une orientation bibliographique sur les sources permet de prendre connaissance des éditions et traductions des textes orientaux évoqués dans l'exposé, et une bibliographie de travaux mise à jour ainsi qu'un index referment l'ouvrage.

Vinciane Pirenne-Delforge (FNRS - Université de Liège)

LABRIQUE Françoise (éd.), Religions méditerranéennes et orientales dans l'Antiquité. Actes du colloque des 23-24 avril 1999, Institut des sciences et techniques de l'Antiquité (UMR 6048), Université de Franche-Comté à Besançon, Le Caire, Institut français d'archéologie orientale, 2002.1 vol. $20 \times 27,5 \mathrm{~cm}$, vII + 243 p. (Bibliothèque d'étude, 135). ISBN : 2-7247-0314-6.

Centrée sur les questions d'interaction culturelle dans les polythéismes antiques, la rencontre à l'origine de ce livre réunissait très logiquement des spécialistes d'horizons divers ( $c f$. le sommaire dans Kemos 16 [2003], p. 393). Le résultat est composite et inégal, sans réelle perspective d'ensemble sur la problématique envisagée ni même une introduction ou une conclusion qui en tiendrait lieu. Qu'on me permette dès lors de privilégier les quelques études qui touchent aux questions religieuses grecques. Alain Blomart reprend le dossier très fréquenté de l'implantation du culte de la Mère des dieux à Athènes et le met en perspective - c'est l'originalité de la réflexion - avec celui d'Apollon Patrôos. Il dessine ainsi le contexte plus large d'une crise d'identité athénienne dans les années 410 et des enjeux politiques de l'Ionie. Il est toutefois délicat d'interpréter Apollon Patrôos comme un "étranger " alors qu'il est explicitement donné comme le père des Athéniens et des Ioniens. - Michèle Broze et Carine Van Liefferinge se penchent ensemble sur le prologue du De Mysteris de Jamblique et montrent avec finesse comment, autour de la figure divine de Thot-Hermès, cette réflexion néoplatonicienne "ancre sa pensée dans les pratiques intellectuelles et rituelles de l'Égypte et de la Grèce, en conciliant les spécificités des deux traditions " (p. 44). - Colette Jourdain-Annequin, dont la thèse sur Héraclès (Héraclès aux portes du soir; Paris, 1989) est un passage obligé pour quiconque étudie le héros/dieu, revient sur l'origine égyptienne qui lui est attribuée par Hérodote. Elle 'piste' les interpretationes du même type dans la tradition grecque en les mettant en regard des études modernes sur les différents "syncrétismes » qui ont affecté sa figure (phénicien, chypriote). - Eric Le Berre analyse la manière dont Hérodote, dans l'épisode de la vengeance du taureau Apis contre Cambyse, insiste sur la nature divine de l'animal 